\title{
Stufenmodell der Normalitätsreflexionen. Wie Lehrpersonen kulturelle Heterogenität in Schule und Unterricht unterschiedlich reflektieren
}

\section{Bruno Leutwyler, Eveline Steinger und Priska Sieber}

Mit der Selbstverständlichkeit kultureller Heterogenität in Gesellschaft, Schule und Unterricht sieht sich die Lehrerinnen- und Lehrerbildung herausgefordert, Lehrpersonen den Erwerb von Fähigkeiten zu ermöglichen, die eine produktive Gestaltung von Schule und Unterricht im multikulturellen Kontext erlauben. Bisherige Modelle, die den Erwerb interkultureller Fähigkeiten konzeptualisieren, fokussieren allerdings zu wenig auf den spezifischen Kontext der monokulturell geprägten Institution Schule. Das in diesem Beitrag vorgestellte Forschungsprojekt arbeitet deshalb prototypische Reflexionsmuster heraus, wie (angehende) Lehrpersonen kulturelle Unterschiede spezifisch im schulischen Kontext reflektieren, und verdichtet diese Reflexionsmuster zu einem Stufenmodell der Normalitätsreflexionen. Damit werden unterschiedliche Reflexionstiefen bezüglich kultureller Unterschiede im monokulturell geprägten schulischen Kontext beschrieben, die in der Lehrerinnen-und Lehrerbildung diagnostiziert und gezielt bearbeitet werden können.

\section{Einleitung}

Die zunehmende weltweite Migration sowie die Individualisierung unserer Gesellschaft während der letzen Jahrzehnte zeigen weitreichende Folgen für Schule und Unterricht. Die kulturelle Heterogenität der an der Schule Beteiligten hat zugenommen und damit auch die Anforderungen an Lehrpersonen, mit kulturellen Unterschieden umzugehen. Für die Lehrerinnen- und Lehrerbildung stellt sich damit die Aufgabe, den (angehenden) Lehrpersonen den Erwerb von Fähigkeiten zu ermöglichen, mit kultureller Heterogenität bei der Gestaltung von Schule und Unterricht produktiv umgehen zu können. Bevor allerdings ein Konzept zum Erwerb entsprechender Fähigkeiten beschrieben werden kann, muss Klarheit darüber geschaffen werden, welche in der Lehrerinnen- und Lehrerbildung bearbeitbaren Fähigkeiten denn zu einem optimalen Umgang mit kultureller Heterogenität führen. Der vorliegende Beitrag stellt die Entwicklung eines Modells dar, das wesentliche Voraussetzungen für Lehrpersonen für den produktiven Umgang mit kultureller Heterogenität in Schule und Unterricht beschreibt. 
Ausgangslage dafür stellt im nächsten Abschnitt eine Übersicht über bestehende Modelle dar, die den Fähigkeitserwerb im Bereich interkultureller Begegnungssituationen konzeptualisieren. Dabei wird deutlich, dass in all diesen Modellen der Reflexion ein zentraler Stellenwert beigemessen wird, dass dabei jedoch die Eigenheiten des schulischen Kontextes nicht angemessen berücksichtigt werden. Deshalb spezifiziert ein weiterer Abschnitt, inwiefern ein angemessener Umgang mit kultureller Heterogenität innerhalb der monokulturell geprägten Institution Schule auch Gestaltungsformen von Schule und Unterricht als kulturell geprägte Normalitätsvorstellungen erfassen sollte. Im darauf folgenden Abschnitt wird die methodische Anlage eines Forschungsprojektes beschrieben, welches die Reflexionstiefe angehender Lehrpersonen bezüglich kultureller Heterogenität im spezifischen Kontext der Schule erfasst. Im Ergebnisteil werden anschliessend vier prototypische Reflexionsmuster beschrieben und zu einem Stufenmodell der Normalitätsreflexionen verdichtet, das unterschiedliche Reflexionstiefen bezüglich kulturell geprägter schulischer Praktiken darstellt. In einem abschliessenden Abschnitt wird schliesslich das neu entwickelte Stufenmodell mit bestehenden Modellen des Fähigkeitserwerbs im Bereich interkultureller Begegnungssituationen verglichen und sein Potential für die Förderung interkultureller Fähigkeiten in der der Lehrerinnen- und Lehrerbildung diskutiert.

\section{Stufenmodelle des Fähigkeitserwerbs}

In der Literatur findet sich eine Vielzahl von Modellen, die - um sie zunächst sehr verallgemeinernd auf einen Nenner zu bringen - notwendige Fähigkeiten oder Kompetenzen beschreiben, um mit Menschen aus anderen Kulturen erfolgreich kommunizieren zu können. Als gemeinsames Merkmal dieser Modelle wird die Prozesshaftigkeit des Fähigkeitserwerbs betont, welche im Sinne eines Stufenmodells einen Ausgangs- und einen Zielzustand beschreibt (Ringeisen, Buchwald \& Schwarzer, 2007). Idealtypisch wird diese Vorstellung eines gestuften Entwicklungsprozesses in den folgenden vier breit rezipierten Modellen deutlich:

- Eine klassische Konzeption ist etwa im «Stufenmodell interkulturellen Lernens» von Hoopes (1979) realisiert. In sechs Stufen soll nach diesem Ansatz die Entwicklung interkultureller Kompetenz von der Sensibilisierung für die Problematik über die kulturelle Selbstreflexion zur kulturellen Fremdreflexion führen und von dort zur Überprüfung dieser Reflexion in oder an der Realität einer Zielkultur. Dazu beschreibt Hoopes (1979) die sechs Stufen als (1) Überwindung des «natürlichen Ethnozentrismus», (2) "Aufmerksamwerden für Fremdes», (3) «Verständnis», (4) «Akzeptieren anderer Kulturen so wie sie sind», (5) «Bewertung und Beurteilung» und schließlich (6) «selektive Aneignung» von Kultur, womit eine «interkulturelle Akkulturation» erreicht wird. 
- Bennett (1986) postuliert auf einer sozialkonstruktivistischen Grundlage in seinem "Developmental Model of Intercultural Sensitivity " eine Abfolge von sechs Stadien mit je zunehmender Bewusstheit kultureller Unterschiede. Dieses Modell geht davon aus, dass interkulturelle Sensibilität mit einer zunehmenden Vielfältigkeit an Erfahrungen mit kulturellen Differenzen wächst, dass also die Fähigkeit zu professionellem Handeln in anspruchsvollen interkulturellen Situationen zunimmt, wenn das Bewusstsein für kulturelle Unterschiede wächst und somit die Sensibilität für kulturelle Prägungen und Wahrnehmungen grösser wird. Die ersten drei Stadien innerhalb dieses Modells sind ethnozentrische Stadien, wobei (1) kulturelle Unterschiede zunächst verneint («Denial»), in der Folge (2) abgewehrt oder negativ bewertet («Defense») und schliesslich (3) banalisiert werden («Minimization»). Nach diesen drei ethnozentrischen Stufen folgt die idealtypische Entwicklung in weiteren drei ethnorelativen Stadien, wobei (4) eine Anerkennung und Wertschätzung kultureller Unterschiede ("Acceptance») sowie (5) ein beweglicher Bezugsrahmen als Voraussetzung für Empathie entwickelt werden («Adaptation») und schliesslich (6) die Erfahrung einer pluralistischen Weltsicht zu einer neuen Identität integriert wird («Intergration»).

- Ein weiteres, breit rezipiertes Modell ist das "Handlungs- und lerntheoretische Konzept zur Entwicklung interkultureller Kompetenz» von Thomas (2003). Thomas definiert interkulturelle Kompetenz als die «Fähigkeit, kulturelle Bedingungen und Einflussfaktoren im Wahrnehmen, Urteilen, Empfinden und Handeln bei sich selbst und bei anderen Personen zu erfassen, zu respektieren, zu würdigen und produktiv zu nutzen im Sinne einer wechselseitigen Anpassung, von Toleranz gegenüber Inkompatibilitäten und einer Entwicklung hin zu synergieträchtigen Formen der Zusammenarbeit, des Zusammenlebens und handlungswirksamer Orientierungsmuster in Bezug auf Weltinterpretation und Weltgestaltung»(S. 143). In seinem Konzept zur Entwicklung interkultureller Kompetenz geht der interkulturelle Lernprozess von personalen Grundbedingungen aus, beginnt im Vollzug interkultureller Erfahrungen und führt dann über die Lernstufen interkulturelles Lernen, interkulturelles Verstehen bis hin zur Entwicklung einer handlungswirksamen interkulturellen Kompetenz. Nach Thomas (ebd.) sind dabei u.a. «der gezielte Einsatz von Reflexion und Kommunikation über das, was an situationsund zielangemessenem Verhalten zu planen, durchzuführen und als Ergebnis zu registrieren und zu bewerten ist, zentrale Anforderungen» (S. 143).

- Und schliesslich präsentiert Gogolin (2003) das spezifisch auf den Schulbereich ausgerichtete Modell der «Fähigkeitsstufen der Interkulturellen Bildung». Es benennt sieben übergreifende, aufeinander aufbauende Fähigkeitsstufen, die durch interkulturelle Bildung und Erziehung erreicht werden können: (1) Kenntnisse über Phänomene, in denen sich kulturelle, sprachliche oder soziale Verschiedenheit zeigt, (2) Kenntnisse über Gründe und Anlässe für diese Phänomene ${ }^{1}$, (3) Fähigkeit, diese Phänomene in ihrem Wandel zu be- 
trachten und zu reflektieren, (4) Fähigkeiten, diese Phänomene aus verschiedenen Perspektiven zu betrachten und zu reflektieren, (5) Fähigkeit zur Durchdringung und Verknüpfung historischer, politischer und gesellschaftlicher Zusammenhänge, aufgrund derer Phänomene für das Leben eines Menschen oder einer Gruppe bedeutsam werden, (6) Fähigkeit, die eigene Wahrnehmung, Empfindung und Verhaltensgewohnheiten bei der Begegnung mit Fremden oder Fremden zu erkennen und zu verstehen, worauf sie jeweils zurückzuführen sind, (7) Fähigkeit, das eigene Handeln und Verhalten, die eigenen Gewohnheiten und Wertorientierungen an den moralischen und ethischen Standards einer modernen, pluralen, weltoffenen und demokratischen Gesellschaft auszurichten.

Neben dem stufenartigen Aufbau ist diesen Modellen gemeinsam, dass Fremdes, Andersartigkeit bzw. Verschiedenheit zunächst einer Aufmerksamkeit bedarf (Hoopes, 1979), erkannt bzw. «recognised "(Bennett, 1986), wahrgenommen bzw. erfasst (Thomas, 2003) werden muss oder Kenntnisse davon aufgebaut (Gogolin, 2003) werden müssen. In diesen Modellen wird weiter eine Stufe des $A k-$ zeptierens (Hoopes, 1979), von "acceptance " (Bennett, 1986) oder Respektieren (Thomas, 2003) formuliert, wobei Gogolin (2003) dies nicht als Voraussetzung für interkulturelle Kompetenz beschreibt, sondern die Fähigkeit zur Begründung eigener Wahrnehmung, Empfindung und Verhaltensgewohnheiten bei der Begegnung mit Fremden als bedeutsam erachtet. Das Modell von Gogolin ist denn auch das Einzige, das nicht explizit affektive oder emotionale Aspekte thematisiert. Schliesslich verlangen alle Modelle im Rahmen des interkulturellen Fähigkeitserwerbs von den Lernenden eine selektive Aneignung (Hoopes, 1979), eine "adaptation» und "integration" (Bennett, 1986), bzw. eine wechselseitige Anpassung (Thomas, 2003) oder das eigene Handeln und Verhalten an den moralischen und ethischen Standards einer modernen Gesellschaft auszurichten (Gogolin, 2003): Fähigkeiten also, die in der Lehrerinnen- und Lehrerbildung als Berufsbildung für Lehrpersonen in multikulturellen Gesellschaften zentral zu fördern sind, deren Veränderung allerdings nur bedingt empirisch erfassbar ist.

\section{Die Bedeutung der Reflexion}

Eine weitere Gemeinsamkeit dieser Modelle ist der zentrale Stellenwert der Reflexion für den Fähigkeitsaufbau. ${ }^{2}$ So formulieren denn auch Karakasoglu und Bandorski (2008) in ihrem Zeitschrifteneditorial zu «Interkultureller Bildung»: «Kernbestandteil der so zu entwickelnden interkulturellen Kompetenz ist eine reflektierte Position» (S. 2), wobei für Lehrpersonen «eine kritische Reflexion des institutionellen und professionellen Selbstverständnisses» (ebd., S. 5) von besonderer Bedeutung sei. Allerdings bleibt der Begriff der Reflexion in allen präsentierten Modellen eher vage, so auch generell in der Lehrerinnen- und Lehrerbil- 
dung, obwohl Reflexion dort als allgegenwärtig gelten kann. Wird Reflexion als der zentrale Methodenbegriff der neueren Philosophie - für die Sozialwissenschaften fruchtbar gemacht und entlang von Ulfig (1997) etwa verstanden als die "Struktur der Thematisierungsweise», kann jedoch erwartet werden, dass der Umgang mit kulturellen Unterschieden zumindest auf einer kognitiven Ebene erfasst werden kann: als Art und Weise, wie kulturelle Unterschiede beschrieben, strukturiert, erklärt oder begründet werden. Entsprechende Beschreibungen, Strukturierungen, Erklärungen oder Begründungen setzen ein bestimmtes Thematisierungsvermögen voraus (ebd., S. 11) und können deshalb als Ausdrucksform der Tiefe und der Differenziertheit von Reflexion verstanden werden.

Das Thematisierungsvermögen von kulturellen Unterschieden wird im vorliegenden Beitrag bezüglich eines psychologisch und kulturanthropologisch verankerten Kulturbegriffs betrachtet. Kultur wird entsprechend konzeptualisiert als von einzelnen Handelnden in spezifischen alltäglichen Interaktionen erworbene je unterschiedliche Wissensordnungen, Denkstrukturen und Wertvorstellungen sowie Komplexe sozialer Praktiken und Verhaltensstrukturen (Reckwitz, 2001). Sozialen Phänomenen, wie sie etwa in schulischen Kontexten zu beobachten sind, liegen kollektive Repräsentationen zugrunde, die jedoch oft nicht ins Bewusstsein der Handelnden rücken. Damit wird das in diesem Beitrag erforschte Thematisierungsvermögen konzeptualisiert als die Fähigkeit, unterschiedliche Wissensordnungen, Deutungsstrukturen, Wertvorstellungen, Praktiken und Verhaltensformen möglichst konsistent und situationsspezifisch genau (BenderSzymanski, Hesse \& Göbel, 2000) beschreiben, strukturieren, erklären und begründen zu können.

Eine differenzierte Beschreibung dieses Thematisierungsvermögens bezüglich kultureller Unterschiede im schulischen Kontext ist in diesem so verstandenen Sinn mit den oben beschriebenen Modellen des Fähigkeitserwerbes nicht möglich. Diese Modelle ermöglichen deshalb auch keine Beschreibung, welche Art von Reflexionen spezifisch für angehende Lehrpersonen in den Institutionen der Lehrerinnen- und Lehrerbildung zu kultivieren, zu unterstützen und zu fördern wären. So sind - mit Ausnahme des Modells von Gogolin (2003) - alle bisher entwickelten Beschreibungen von Fähigkeitsstufen im Bezug zu einem kulturell heterogenen Umfeld nicht spezifisch auf den schulischen Kontext ausgerichtet, sondern befassen sich mit Fähigkeiten und Kompetenzen für erfolgreiche interkulturelle Kommunikation im Allgemeinen. Aber auch das gogolinsche Modell, das sich auf «interkulturelle Bildung» bezieht, formuliert Ergebnisstufen einer solchen Bildung für jegliche Zielgruppen und ist entsprechend nicht auf besondere Fähigkeiten von Lehrpersonen ausgerichtet. Im kulturell heterogenen schulischen Kontext sind jedoch weitere oder andere Fähigkeiten für Lehrpersonen von Bedeutung als in solch allgemeinen Modellen erfasst, da gleiches Handeln in unterschiedlichen kulturellen Kontexten völlig verschiedene Folgen zeigen kann (Jahoda, 1996). 


\section{Aspekte der Reflexion: Kulturelle Heterogenität in der monokulturellen Institution Schule}

Zu den Eigenheiten, die es spezifisch im schulischen Kontext zu reflektieren gilt, gehört das monokulturell geprägte Selbstverständnis der Institution Schule (Gogolin, 1994). Eine vertiefte Reflexion über kulturelle Unterschiede in der Institution Schule, die sowohl Ausdruck von Kultur ist als auch den Auftrag hat, Kultur zu vermitteln (Bruner, 1996), kann sich nicht auf eine Auseinandersetzung mit individuellen kulturellen Besonderheiten beschränken. Vielmehr haben Lehrpersonen ihre eigene, oft unbewussten Deutungsstrukturen, Wertvorstellungen und Praktiken im Kontext der Institution «Schule» mit zu berücksichtigen, wenn sie Aspekte kultureller Heterogenität reflektieren. Dabei ist zu beachten, dass die Pflichtschule, wie sie vor rund zweihundert Jahren als wichtiges Teilsystem moderner Gesellschaften eingerichtet wurde, nicht zuletzt auch zur Konsolidierung einer inneren kulturellen Einheit in einer Gemeinschaft beitragen sollte und so Monokulturalität Ziel und Programm von Schulbildung wurde (Radtke, 2004, S. 630). Das monokulturelle Selbstverständnis der heutigen multikulturellen Schule (Gogolin, 1994) ist deshalb auch Teil und Ausdruck eines in der Moderne geprägten Verhältnisses zu Normen und zu Normalität, deren Archäologie beispielsweise Foucault beschreibt. In dieser Perspektive bildet die Normierung und Normalisierung des Individuums - im Kontext der Schule: der Schülerschaft und des Lehrpersonals - ein zentrales Charakteristikum moderner Gesellschaften (Foucault, 1973, 1976). So kann etwa die Etablierung von Jahrgangsklassen, in welchen Kinder in Klassen von «Gleichen» eingeteilt werden und sich mit dem Gleichen beschäftigen, frontal vor dem Lehrer und dessen «überwachendem Blick» (Foucault, 1976, S. 221ff.) aufgereiht, als Ausdruck von Normalisierungsbestrebungen interpretiert werden, die das Massnehmen an einheitlichen Normen - seien dies Leistungs-, Verhaltens- oder zeitliche Normen etablieren.

In einem multikulturellen schulischen Kontext stossen allerdings verschiedene kulturelle Bezugssysteme mit je unterschiedlichen Werten und Normen und also auch mit unterschiedlichen Vorstellungen davon, was als «normal» erachtet wird - aufeinander. In diesem Sinne treffen unterschiedliche Normalitäten aufeinander. Die in einer Schule gelebte Normalität kann dabei als Mass des konsensfähigen Miteinanders verstanden werden, wie es Link (2006) in der ersten fundierten Normalismus-Theorie vorschlägt. Eine so verstandene Normalität ist deshalb in pluralistischen und demokratischen Gesellschaften als flexibel zu betrachten, die von einer überwiegenden Mehrzahl der Teilnehmenden einer Gesellschaft sozial konstruiert wird. Eine elaborierte Reflexion kultureller Unterschiede im Kontext kultureller Vielfalt bedarf deshalb auch einer bewussten Wahrnehmung kulturell geprägter Normalitäts-Vorstellungen, wie sie in der Schule als normalisierender Institution eingeübt wurden und nach wie vor werden. Wenn also (angehende) Lehrpersonen über kulturelle Heterogenität im Kontext Schule 
reflektieren, stellt sich auch die Frage, in welchem Ausmass sie mehr oder weniger enge Vorstellungen von Normalität erkennen und als spezifische Ausdrucksform einer bestimmten kulturellen Praxis interpretieren können. Erst mit der Reflexion dieser Zusammenhänge kann der bereits oben formulierte Anspruch von Karakasoglu und Bandorski (2008) an Lehrpersonen, "eine kritische Reflexion des institutionellen und professionellen Selbstverständnisses» (S. 5) zu leisten, eingelöst werden.

Insofern kann gefolgert werden, dass ein empirisch fundiertes Modell fehlt, das eine differenzierte Beschreibung der Reflexionstiefe, des Thematisierungsvermögens bezüglich kultureller Unterschiede im schulischen Kontext erlauben würde. An diesem Punkt setzt das im vorliegenden Beitrag vorgestellte Forschungsprojekt an. Es geht der Frage nach, wie (angehende) Lehrpersonen kulturelle Unterschiede im schulischen Kontext reflektieren und in welchem Ausmass sie die Dimension «Kultur» bei der Wahrnehmung von Gestaltungsformen von Schule und Unterricht berücksichtigen, wie weit sie also eine Sensibilität für kulturell geprägte Normalitätsvorstellungen entwickelt haben. Eine Antwort auf diese Fragen verspricht einen wesentlichen Beitrag zu leisten, wie in den Institutionen der Lehrerinnen- und Lehrerbildung die Vorbereitung angehender Lehrpersonen auf einen produktiven Umgang mit kultureller Heterogenität unterstützt und optimiert werden kann.

Um diese Fragestellungen zu beantworten, wurde ein offener, explorativer $\mathrm{Zu}$ gang gewählt, wie er der Methodologie der Grounded Theory entspricht (Corbin \& Strauss, 1990; Strauss, 1991). Die empirische Basis für diesen Zugang sowie methodologische Überlegungen werden im folgenden Kapitel skizziert.

\section{Methode}

Ausgangslage bildete eine Befragung von Studierenden der Pädagogischen Hochschule Zentralschweiz (PHZ), die im Rahmen ihrer Ausbildung zur Lehrperson einen Mobilitätsaufenthalt absolviert hatten. Weil Studierende, die in einem fremdkulturellen Kontext eine gewisse Zeit studieren oder ein Schulpraktikum absolvieren, unterschiedliche Gestaltungsformen von Schule und Unterricht unmittelbar erleben, können diese gezielt darüber Auskunft geben, inwiefern sie überhaupt entsprechende Unterschiede wahrnehmen, wie sie allfällige Unterschiede reflektieren und welchen Stellenwert sie der Dimension «Kultur» bei der Erklärung dieser Unterschiede beimessen. Deshalb wurden aufgrund von Schlussberichten, in welchen alle Mobilitätsstudierenden der PHZ nach ihrer Rückkehr über ihren Aufenthalt berichten und berufsrelevante Lernprozesse beschreiben, 17 besonders aussagekräftige Fälle ausgewählt. Mit diesen 17 ausgewählten Studierenden wurden halbstrukturierte Interviews geführt, so dass die interviewten Studierenden einerseits frei über ihre Erfahrungen während des Mobilitätsaufenthaltes berichten und andererseits gezielt über die Wahrneh- 
mung von Unterschieden und den Stellenwert der Dimension «Kultur» bei der Erklärung von Unterschieden reflektieren konnten. Je zwei Studierende wurden zu einem 4-wöchigen Praktikumsaufenthalt in Australien, Frankreich oder Mazedonien befragt, $11 \mathrm{zu}$ einem Semesteraufenthalt in Australien, Dänemark, England, Finnland, Frankreich oder Norwegen.

Im Sinne der Grounded Theory wurde die Analyse dieser 17 Erzählungen nicht als linearer Prozess verstanden, bei dem das zu untersuchende Feld vor dem Eintritt bereits konstruiert wird, sondern vielmehr als ein interpretativer Prozess, der den Daten mehr Bedeutung beimisst als den theoretischen Vorannahmen. Ein solcher Analyseprozess zielt auf eine Strukturierung des Datenmaterials in intensiver Interaktion zwischen Datenmaterial, theoretischen Konzepten und analytischer Interpretation. Damit wird eine empiriebegründete Strukturierung möglich, die dem Datenmaterial neue «konzeptuelle Etiketten» (Corbin, 2003, S. 71) abzugewinnen und «emergierende Konzepte» (ebd.) zu benennen vermag. Eine Generalisierung solcher emergierender Konzepte beruht deshalb nicht auf statistischer Repräsentativität, sondern auf theoretischer Plausibilität und auf konzeptionell repräsentativen Daten.

Im vorliegenden Forschungsprojekt wurden konzeptionell repräsentative Daten durch theoretisches Sampling angestrebt, wie es dem prototypischen Zugang der Grounded Theory entspricht (Corbin, 2003, S. 72). In einem ersten Schritt wurde ein theoretisches Sampling innerhalb des umfangreichen Datenmaterials aus den 17 mündlichen Erzählungen ehemaliger Mobilitätsstudierender angewendet, das genügend vielfältig und reichhaltig war, um sequentiell zu analysieren und gezielt kontrastierende Fälle einzubeziehen. Erst in einem zweiten Schritt wurden gezielt weitere Studierende der PHZ sowie Fachpersonen aus den Bereichen «Interkulturelle Pädagogik» und «Migration und Bildung» befragt, um die emergierenden Konzepte aus der ersten Analysephase zu überprüfen und zu differenzieren. Die Befragungen in dieser zweiten Phase fokussierten deshalb auch thematisch gezielter auf die Dimension «Kultur» bei der Erklärung von Unterschieden und wurden nicht mehr an Erfahrungen in einem Mobilitätsaufenthalt geknüpft. So wurden beispielsweise Fragen gestellt wie «Bitte beschreiben Sie Ihre Vorstellung, inwiefern der kulturelle Hintergrund von Schülerinnen und Schülern einen Einfluss auf deren Lernen hat.» Mit solchen Fragen wurden die Befragten explizit aufgefordert, die kulturelle Dimension zu reflektieren. Gerade weil die Befragten damit auf kulturelle Aspekte eingespurt wurden, konnte in den entsprechenden Aussagen identifiziert werden, wie differenziert unterschiedliche Wissensordnungen, Deutungsstrukturen, Wertvorstellungen, Praktiken und Verhaltensformen erkannt, beschrieben, strukturiert, erklärt und begründet werden. Damit erlaubt dieser Zugang die Identifikation unterschiedlicher Tiefe und Differenziertheit der Reflexion und damit eine Identifikation des Thematisierungsvermögens kultureller Unterschiede.

Beim Zugang der Grounded Theory gilt eine Analyse dann als saturiert, wenn im kontinuierlichen Mäandrieren zwischen Datenmaterial und emergie- 
renden Konzepten keine Widersprüche entstehen, sich keine neuen Aspekte zu den Konzepten ergeben und diese deshalb im Datenmaterial verankert sind (Corbin, 2003, S. 74). Das geschilderte zweistufige Vorgehen im vorliegenden Projekt ermöglichte eine Analyse von reichhaltigem Datenmaterial, welches als konzeptionell repräsentativ gelten darf und das keine Widersprüche zu den emergierenden Konzepten enthält, wie sie nun im folgenden Abschnitt vorgestellt werden.

\section{Ergebnisse: Prototypische Reflexionsmuster}

Die Art und Weise, wie angehende Lehrpersonen kulturelle Heterogenität im schulischen Kontext reflektieren, unterscheidet sich beträchtlich. Aus der Vielfalt an Überlegungen, die im reichhaltigen Datenmaterial sichtbar wurden, emergierten schliesslich vier prototypische Reflexionsmuster bei der Wahrnehmung und Reflexion kultureller Unterschiede und bei der Sensibilität für kulturell geprägte Normalitätsvorstellungen:

Kulturblinde Reflexion: Erstens gibt es eine Gruppe von angehenden Lehrpersonen, die Kultur im schulischen Kontext kaum wahrnehmen, oder wenn sie doch kulturelle Unterschiede wahrnehmen, diese nicht in einem kulturellen Kontext reflektieren. Diese Studierenden antworten auf die Frage, inwiefern der kulturelle Hintergrund von Schülerinnen und Schülern einen Einfluss auf deren Lernen hat, ohne Bezug zu einer kulturellen Dimension: "Ich habe das in der Primarschule so erlebt, dass die meisten Migrationskinder eher schlechtere Schüler waren. Ich weiss nicht, ob sie es nicht verstanden haben wegen der Sprache oder ob sie zwar sprachlich alles verstanden haben, aber im Gehirn nicht folgen konnten.» In dieser Aussage wird zwar ein Unterschied benannt, jedoch pauschalisierend, undifferenziert und ohne Erklärungsansätze, die kulturelle Phänomene jenseits der Sprachkenntnisse berücksichtigen würden. Sogar Sprache wird nicht als kulturelle Dimension im Sinne einer Ausdrucksgebung für unterschiedliche Wissensordnungen und Deutungsstrukturen verstanden. Entsprechend werden Unterschiede, die nicht mit dem geäusserten Verständnis von Sprache erklärt werden können, auf personalisierte Merkmale wie etwa das individuelle Auffassungsvermögen bezogen. Eine vergleichbare Nicht-Berücksichtigung kultureller Merkmale zeigt sich auch in folgender Aussage einer anderen Studentin: «Ich sehe, dass Lernumfelder von Migrantenschülern oft anders sind als diejenige von Schweizern. Das Lernen wird somit indirekt über die Lernsituation zuhause beeinflusst. Hat das Lernen z.B. für die Eltern eines Migrationskindes einen geringen Stellenwert, wird nicht die nötige Ruhe und Konzentration vorhanden sein, um das Lernen entsprechend zu fördern. Auch beinhaltet ein Lernumfeld die in der Schule gesprochene Sprache, was zu Hause oft nicht der Fall ist.» Mit dem Hinweis auf den Stellenwert des Lernens bei den Eltern wird zwar auch in diesem Fall ein Unterschied wahrgenommen und als kulturel- 
ler Unterschied dargestellt. Die Reflexion dieses Unterschieds berücksichtigt allerdings nur vordergründig eine kulturelle Dimension: Es werden Erklärungsansätze postuliert, die zwar durchaus mit unterschiedlichen Deutungsmustern und Wertvorstellungen in einem Zusammenhang stehen können. Die pauschalisierende und undifferenzierte Form der Aussage lässt jedoch darauf schliessen, dass hier keine situationsspezifische Wahrnehmung unterschiedlicher Deutungsmuster erfolgt und vielmehr stereotypische Aspekte benannt werden. Das Ausblenden kultureller Dimensionen wird schliesslich bei einer weiteren prototypischen Aussage deutlich, die allerdings Verständnis für fremdkulturelle Schülerinnen und Schüler suggeriert: «Ich denke der grösste Unterschied macht die Sprache, wenn das Kind eine andere Sprache spricht, kann das sein Lernen verlangsamen und es braucht mehr Unterstützung als andere Kinder. Aber Kinder passen sich in der Regel recht schnell an, darum denke ich, hat ein Kulturunterschied keinen grossen Einfluss.» Diese Aussage signalisiert zwar eine gewisse $\mathrm{Zu}-$ gewandtheit zu interkulturellen Fragen, die Reflexion der Unterschiede bleibt jedoch ebenfalls sehr undifferenziert und beschränkt sich auf die Sprache als einziges Unterscheidungsmerkmal von Kultur, wobei auch hier Sprache nicht als Ausdruck unterschiedlicher Deutungsstrukturen interpretiert und somit die kulturelle Dimension von Sprache nicht benannt wird. Die Bedeutung einer kulturgebundenen Unterschiedlichkeit wird gar explizit negiert. In einem solchen Reflexionsmuster, das die Dimension Kultur im schulischen Kontext kaum berücksichtigt, kann gar keine Vorstellung von Normalität hinterfragt werden, weil Schul-, Unterrichts- und Lernprozesse nicht als Ausdrucksformen einer spezifischen Vorstellung von Normalität erkannt werden.

Kulturgebundene Reflexion: Bei einem zweiten Reflexionsmuster werden kulturelle Aspekte differenzierter mit einbezogen, wenn kulturelle Heterogenität im schulischen Kontext reflektiert wird. So weist die folgende Antwort auf die Frage, was eine angehende Lehrperson noch lernen sollte, um optimal auf den Umgang mit kulturell heterogenen Klassen vorbereitet zu sein, auf kulturelle Aspekte hin. Diese kulturellen Aspekte werden jedoch innerhalb einer eigenen kulturellen Vorstellung reflektiert, so dass die Reflexion von Unterschieden auf eine kulturgebundene Verarbeitung hinweist: «Ich glaube, dass es wichtig ist, die verschiedenen Religionen zu kennen. So kann ich entscheiden, wann ich auf einer bestimmten Tätigkeit bestehen kann und wann ich mich aufgrund der Religion fügen muss. Ich bin der festen Überzeugung, dass es kulturelle Schwierigkeiten gibt, auf welche man als Lehrperson aber nicht immer in Form einer Anpassung reagieren muss. Die Anpassung muss gegenseitig erfolgen.» Mit dem Hinweis auf die Notwendigkeit einer gegenseitigen Anpassung wird eine gewisse Offenheit für kulturelle Aspekte in schulischen Lern- und Entwicklungsprozessen signalisiert und Unterschiede werden durchaus als kulturbedingt wahrgenommen. Die wenig differenzierte Argumentation, sich bei denjenigen kulturellen Unterschieden zu «fügen», die auf religiöse Hintergründe zurückzuführen sind, verweist allerdings auf einen klaren Referenzrahmen, der vorgeben kann, in 
welcher Situation sich wer wem anzupassen hat. Eine reflektierte Begründung dafür bleibt aus, eine Vorstellung davon, dass auch religiöse Phänomene durchaus sehr unterschiedlich wahrgenommen, gedeutet und interpretiert werden können, findet sich in der vorliegenden Aussage ebenso wenig. Das Fehlen einer problematisierenden Auseinandersetzung mit gegenseitiger Perspektivenübernahme deutet auf klare Vorstellungen hin, welche Regeln gelten und wie diese hierarchisch zueinander stehen. Religion und Kultur werden praktisch synonym gesetzt, eine Auseinandersetzung mit den Zusammenhängen dieser beiden Phänomene wird nicht ersichtlich. Die gleichermassen an eigenen kulturellen Bezugssystemen orientierte Reflexion wird auch in folgender Aussage einer anderen Studentin deutlich: "Ich muss mich mit verschiedenen Kulturen vertraut machen, muss wissen, was legitim ist und was ev. von den Eltern so ausgelegt wird.» Auch bei dieser Aussage wird deutlich, dass die Dimension Kultur in der Reflexion sehr wohl berücksichtigt wird. Der Wunsch zu erfahren, was kulturell legitim ist, und dies als Gegensatz zu Auslegungsmöglichkeiten von Eltern zu deuten, weist allerdings darauf hin, dass die eigene Deutung als Referenz für die Beurteilung von Legitimität gilt und dass deshalb keine kulturelle Perspektivenübernahme erfolgt. Ein analoges Muster zeigt sich in der Aussage einer weiteren Studentin: «Ich möchte noch genauer erfahren, wie man ein Migrantenkind richtig eingliedern kann in eine Klasse, wie man die Kultur des Kindes den anderen Mitschülern aufzeigen kann ohne es ,schlechter' oder halt als ,anders' zu bezeichnen.» Diese Aussage signalisiert eine klare Zuwendung zu Kindern mit einem fremdkulturellen Hintergrund. Das Bemühen «richtig eingliedern» zu können lässt jedoch vermuten, dass ein Eingliedern in Eigenes gemeint ist, dass also weiterhin der eigene kulturelle Hintergrund als Referenzrahmen fungiert, welcher kein kulturrelatives Verständnis enthält. Die bewusst vorsichtige Formulierung signalisiert zwar Sensibilität für einen nicht-wertenden Umgang, die Möglichkeit dazu scheint jedoch noch nicht vorhanden. Zu diesem Reflexionsmuster können auch durchaus differenzierte Aussagen zu kulturellen Aspekten gehören, wenn diese im Kontext eigener Werte und Vorstellungen formuliert werden. Dies zeigt sich beispielsweise in folgender Aussage: «Schülerinnen und Schüler sind stark geprägt vom System und den Werten ihres Herkunftslandes. In vielen Ländern wird selbständiges Lernen/Denken und Hinterfragen den Schülern nicht gelehrt oder sogar verboten! Für solche Menschen ist es vielleicht schwieriger zu hinterfragen, eigenständig zu denken und sich mit anderen auszutauchen. In manchen Ländern haben Frauen nichts zu sagen! Für Schüler (!) aus diesen Ländern ist es oftmals schwer eine Lehrerin zu respektieren.» Die Kulturgebundenheit von gewissen Unterschieden wird hier deutlich und in Bezug zu Werten der Herkunftskultur gesetzt. Zudem wird anhand konkreter Beispiele illustriert, wie diese Unterschiede zum Tragen kommen können. Aussagen wie Frauen hätten «nichts zu sagen» oder selbständiges Denken sei «verboten» deuten jedoch klar darauf hin, dass kulturelle Unterschiede innerhalb eigener Interpretationsmuster gedeutet werden und die Reflexion insofern unhinterfragt an 
die eigenen Werte und Vorstellungen gebunden bleibt. Somit werden kulturelle Unterschiede auch nicht als Ausdruck unterschiedlicher Deutungsstrukturen und Wissensordnungen wahrgenommen: Es gibt keine Hinweise auf ein Bewusstsein für kulturell bedingte Charakteristika eigener Interpretationsmuster, weshalb auch eigene Schul- und Unterrichtspraktiken kaum als Ausdruck bestimmter kultureller Praktiken gedeutet werden können.

Kulturrelative Reflexion: Ein drittes Reflexionsmuster angehender Lehrpersonen weist auf einen kulturrelativen Umgang mit Unterschieden hin. Eine prototypische Aussage dazu ist folgende Antwort auf die Frage, was noch gelernt werden müsste, um als Lehrperson optimal auf den Umgang mit kulturell heterogenen Klassen vorbereitet zu sein: «Ich muss lernen, inwiefern Kinder aus anderen Kulturen anders sind als Einheimische und wie man mit solchen Kindern im Unterricht umgehen soll, so dass das Klassenklima stimmt und sich alle angesprochen und zufrieden fühlen.» Diese angehende Lehrerin ist sich offenbar bewusst, dass es kulturelle Unterschiede jenseits von Sprache und Religion gibt, dass also beispielsweise Denk- und Reaktionsmuster von Kindern kulturell bedingt verschieden sein können, und sie will diese Phänomene vertieft kennen lernen. Mit dem Wunsch, alle anzusprechen, zeigt sie eine kulturrelative Verarbeitung von Unterschieden: Sie will sich aktiv mit den Unterschieden auseinandersetzen und ist bereit, sich nicht nur im Denken sondern auch im Handeln darauf einzulassen. Eine inhaltliche Konkretisierung wird allerdings noch nicht angesprochen. Dennoch wird deutlich, dass allenfalls andere als gewohnte Unterrichtspraktiken in Betracht gezogen werden, um fremdkulturellen Kindern gerecht werden zu können. Damit wird ein Bewusstsein angesprochen, welches gewohnte Schul- und Unterrichtspraktiken als Ausdrucksformen einer spezifischen kulturellen Praxis, einer spezifischen Normalitätsvorstellung auffasst. Das gleiche Muster wird auch in der folgenden Antwort einer anderen Studentin deutlich: «Ich fände es sehr wichtig, wenn wir uns mit verschiedenen (v.a. auch in der Schweiz vertretenen) Kulturen auseinandersetzen würden und uns so ein Bild machen könnten, wie welche Kultur lebt, denkt und auf bestimmte Lebenssituationen reagiert.» In dieser Antwort wird ein weites Verständnis von Kultur artikuliert, das nicht nur direkt wahrnehmbare Unterschiede berücksichtigt, sondern auch Denk- und Deutungsstrukturen, Wissensordnungen, Wertvorstellungen und Verhaltensformen mit einschliesst. Diese angehende Lehrerin drückt damit ein Bewusstsein für weniger offensichtliche Unterschiede aus, und sie will sich ein Bild machen, wie Menschen aus anderen Kulturen denken. Ihre Reflexion von Unterschieden schliesst dadurch den Versuch einer Perspektivenübernahme ein und darf insofern als kulturrelativ gelten. Der Hinweis auf unterschiedliche Reaktionen in bestimmten Lebenssituationen signalisiert zudem ein Bewusstsein dafür, dass bestimmte - für viele vielleicht als «normal» geltende Situationen von anderen unterschiedlich erlebt werden können, dass also auch «normale» Schul- und Unterrichtspraktiken durchaus nicht für alle «normal» sein müssen: dass also Normalitätsvorstellungen kulturell geprägt sind. 
Kulturdynamische Reflexion: Ein viertes Reflexionsmuster schliesslich beinhaltet Überlegungen, die deutlich auf einen kulturrelativen Umgang mit unterschiedlichen Praktiken und Normalitätsvorstellungen hinweisen und darüber hinaus auch je spezifische Entstehungsbedingungen unterschiedlicher Deutungsstrukturen zu verstehen versuchen. So antwortet eine Studentin auf die Frage nach der optimalen Vorbereitung für den Umgang mit kulturell heterogenen Klassen: «Es ist wichtig zu wissen, welche Wertvorstellungen die Kinder aus verschiedenen Kulturen haben. Ich finde es wichtig sich bewusst zu sein, dass jedes Kind anders erzogen ist und zu Hause mit anderen Vorstellungen aufwächst, vor allem wenn sich die Kultur von unserer stark unterscheidet.» Diese Aussage deutet auf ein vertieftes Verständnis von Kultur hin, das auch auf die Dynamik des Entstehens von Unterschieden verweist. Kultur wird dabei als Ergebnis von Prägungen und langfristigen Sozialisationsprozessen verstanden, inhaltliche Konkretisierungen dieser dynamischen Perspektive werden mit dem Hinweis auf die Erziehung angesprochen, aber nicht elaboriert. Eine ähnlich vertiefte und ebenfalls kulturrelative Reflexion von Unterschieden und derer Entstehungsbedingungen zeigt auch folgende Antwort auf die Frage nach der Bedeutung des eigenen kulturellen Hintergrunds für Lehrpersonen: «Ich denke, der kulturelle Hintergrund fliesst ganz unbewusst in den Unterrichtsstil ein. Erklärungen aber auch Sprachwitze beziehen sich auf Hintergründe, die oft nur in der Kultur, in der ich aufgewachsen bin, vorkommen. Die Gefahr liegt natürlich darin, dass ich diesen kulturellen Hintergrund voraussetze und so manchmal auf Unverständnis stosse.» Diese Aussage zeigt erste Ansätze einer Konkretisierung für den kulturrelativen Umgang mit Unterschieden: Diese Studentin weiss um die Kulturgebundenheit des eigenen Handelns, um deren Entstehungsbedingungen und kann dies mit konkreten Beispielen illustrieren.

Diese vier prototypischen Reflexionsmuster, wie sie aus dem reichhaltigen Datenmaterial entwickelt und oben beschrieben wurden, sind nicht nur als Ausdrucksformen für unterschiedliche Reflexionstiefen beim Nachdenken über kulturelle Unterschiede zu interpretieren, sondern auch als Ausdrucksformen unterschiedlicher Reflexionstiefe bezüglich spezifischer schulischer Praktiken, also bezüglich bestimmter Normalitäten.

\section{Stufenmodell der Normalitätreflexionen}

Im Kontext der monokulturell geprägten Institution Schule weisen die vier prototypischen Reflexionsmuster darauf hin, in welchem Ausmass (angehende) Lehrpersonen die Dimension «Kultur» nicht nur bei individuellen Unterschieden zwischen Schülerinnen und Schülern erkennen, sondern auch bei gelebten Praktiken in einem bestimmten kulturellen Kontext - in welchem Ausmass sie also auch schulische Normalitäten als kulturelle Eigenheiten erkennen. Die vier prototypischen Reflexionsmuster können deshalb auch zu einem Stufenmodell der Normalitätsreflexionen verdichtet werden: 
- Stufe O-kulturblinde Reflexion von Schule und Unterricht: Das erste Reflexionsmuster, das Unterschiede im interkulturellen schulischen Kontext nicht beachtet oder aber die Dimension Kultur bei Erklärungsansätzen höchstens pauschalisierend und undifferenziert auf Sprachkenntnisse reduziert, kann als «Stufe 0» bezeichnet werden. Die Perspektive auf schulische Lern- und Entwicklungsprozesse sowie auf Schul- und Unterrichtspraktiken beinhaltet noch keine elaborierte kulturelle Dimension, ein Bewusstsein für kulturell bedingte Charakteristika von eigenen Schul- und Unterrichtspraktiken ist nicht vorhanden. Insofern ist auch kein Bewusstsein vorhanden, dass eigene Wahrnehmungen von eigenen Normalitätsauffassungen beeinflusst werden: Eigene Interpretationsmuster werden als allgemein gültige Interpretationsmuster wahrgenommen.

- Stufe 1 - kulturgebundene Reflexion von Schule und Unterricht. Das Reflexionsmuster der Stufe 1 berücksichtigt durchaus kulturelle Aspekte bei der Wahrnehmung schulischer Lern- und Entwicklungsprozesse, entsprechende Unterschiede werden jedoch innerhalb eigener Interpretationsschemata reflektiert. Die Reflexion bleibt insofern an eigene Werte und Vorstellungen gebunden. Ein Bewusstsein für kulturell bedingte Charakteristika von eigenen Schul- und Unterrichtspraktiken kann auf dieser Stufe 1 durchaus entstehen, die Beurteilung von Fremdem erfolgt allerdings primär anhand der eigenen Überzeugungen und Wertmassstäbe, es erfolgt noch kein Perspektivenwechsel.

- Stufe 2 - kulturrelative Reflexion von Schule und Unterricht: Das Reflexionsmuster der Stufe 2 beinhaltet ein differenziertes Verständnis von Kultur. Insofern beinhaltet die Reflexion auf dieser Stufe 2 die Auseinandersetzung mit anderen Denk- und Deutungsstrukturen und mit anderen Wertvorstellungen und Verhaltensformen und strebt so nach einer Perspektivenübernahme. Lern- und Entwicklungsprozesse werden kulturrelativ thematisiert. Damit geht die Erkenntnis einher, dass eigene Interpretations- und Konstruktionsmuster kulturell inspiriert sind und keine Allgemeingültigkeit beanspruchen dürfen: Es entsteht ein Bewusstsein, dass die eigenen Wahrnehmungen kontextabhängig und durch eigene Normalitätsauffassungen charakterisiert sind.

- Stufe 3 - kulturdynamische Reflexion von Schule und Unterricht: Das Reflexionsmuster der Stufe 3 ist durch eine differenzierte und elaborierte Auseinandersetzung mit kulturellen Unterschieden geprägt, die eine dynamische Komponente beinhaltet und ein aktives Bemühen um Erklärungsversuche für die Entstehung von Unterschieden einbezieht. Auf dieser Stufe 3 werden eigene Überzeugungen und Wertmassstäbe sowie eigene Praktiken bewusst und deren Kulturgebundenheit erkannt. Damit wird ein Bewusstsein entwikkelt, dass und wie unterschiedliche Kontexte eigene und fremde Wahrnehmungen und Konstruktionen beeinflussen. Lern- und Entwicklungsprozesse sowie Gestaltungsformen von Schule und Unterricht werden so im Kontext unterschiedlicher Normalitätsvorstellungen reflektiert. 


\section{Diskussion und Ausblick}

Ein solches Stufenmodell der Normalitätsreflexionen erlaubt eine differenzierte Beschreibung der Reflexionstiefe, also des Thematisierungsvermögens von (angehenden) Lehrpersonen bezüglich kultureller Unterschiede in Schule und Unterricht. Mit der expliziten Beachtung des spezifischen Kontextes der Schule als monokulturelle Institution spezifiziert das hier vorgeschlagene Modell die bestehenden Modelle von Bennett (1986), Hoopes (1979) und Thomas (2003) für den Bildungsbereich und konkretisiert die «Fähigkeitsstufen der Interkulturellen Bildung» von Gogolin (2003) im Hinblick auf die spezifischen Voraussetzungen für Lehrpersonen, um mit kultureller Heterogenität im schulischen Kontext produktiv umgehen zu können. Zudem stellt das Modell eine zusätzliche Differenzierung der von Bender-Szymanski et al. (2000) vorgeschlagenen zwei unterschiedlichen Verarbeitungsmodi bzw. Interkulturalitätsstrategien im Hinblick auf kognitiven Irritationen im Rahmen von interkulturellen Erfahrungen von Lehrpersonen dar.

Das hier vorgeschlagene Stufenmodell der Normalitätsreflexionen stimmt in zentralen Dimensionen mit den vier diskutierten Modellen überein. Damit ist die theoretische Plausibilität gegeben, wie sie auch als wesentliches Gütekriterium in der Grounded Theory verlangt wird (Corbin, 2003, S. 72). So zielt auch das Stufenmodell der Normalitätsreflexionen auf eine Entwicklung von einer ethnozentrischen zu einer zunehmend ethnorelativen Perspektive, und es konzeptualisiert den Fähigkeitserwerb analog zu den bestehenden Modellen als eine Ausdifferenzierung des Kulturkonzeptes: Wird Kultur zunächst kaum oder nur pauschalisierend als Erklärungsdimension für schulische Phänomene wahrgenommen, werden mit fortschreitender Reflexionstiefe, mit fortschreitendem Thematisierungsvermögen verschiedene kulturelle Ausdrucksformen in der Gestaltung von Schul- und Unterrichtsprozessen wahrgenommen und mit unterschiedlichen, kulturell geprägten Lern- und Entwicklungsprozessen in Verbindung gebracht.

Beim vorgeschlagenen Stufenmodell der Normalitätsreflexionen wird die Ausdifferenzierung des Kulturkonzeptes aber - und das ist im Vergleich zu den Modellen von Bennett (1986), Hoopes (1979) und Thomas (2003) eine deutliche Erweiterung - auch zunehmend von individuellen Komponenten (wie Lernund Entwicklungsprozessen) auf institutionelle Komponenten (wie Gestaltungsformen von Schule und Unterricht) ausgeweitet. Erst mit dieser Erweiterung jedoch berücksichtigt die Fähigkeitsentwicklung diejenigen Aspekte, die für Lehrpersonen im beruflichen Kontext von Bedeutung sind. Vor diesem Hintergrund kann das Stufenmodell der Normalitätsreflexionen als angemessene Konzeptualisierung des Thematisierungsvermögens von Lehrpersonen im Bereich kultureller Heterogenität postuliert werden.

Mit der Betonung des Thematisierungsvermögens als Reflexionstiefe weist das vorgeschlagene Modell eine kognitive Ausrichtung auf, die emotionale und 
affektive Dimensionen (wie beispielsweise Angst vor dem Anderen, Interesse und Neugier an Unterschieden) kaum erfasst. Diese Ausklammerung emotionaler und affektiver Dimensionen unterscheidet das vorgeschlagene Stufenmodell von den bereits vorliegenden Modellen und mag nicht unproblematisch erscheinen. Im Kontext der Lehrerinnen- und Lehrerbildung zeigt sich aber gerade diese Besonderheit als Vorteil, weil die kognitiven Aspekte interkultureller Auseinandersetzungen in den vorherrschenden Lernarrangements der Lehrerinnenund Lehrerbildungsinstitutionen besonders gut thematisier- und bearbeitbar sind. Damit werden emotionale und affektive Dimensionen nicht als weniger relevant betrachtet, aber als weniger gut bearbeitbar postuliert. Vor diesem Hintergrund fokussiert das Stufenmodell der Normalitätsreflexionen auf diejenigen wesentlichen Voraussetzungen von Lehrpersonen für einen produktiven Umgang mit kultureller Heterogenität, die innerhalb der Lehrerinnen- und Lehrerbildung explizit förder- und grundsätzlich auch diagnostizierbar sind. So könnte das vorgeschlagene Modell auch als Diagnose- und Fördermodell genutzt werden, um das Thematisierungsvermögen von (angehenden) Lehrpersonen zu erkennen und gezielt zu unterstützen. Besonders geeignet sind dazu Gesprächsoder Schreibanlässe, wie sie beispielsweise in Anlehnung an den Intercultural Awareness Questionnaire von Ponterotto (Wang \& Meyers, 2007) formuliert werden können: «Bitte beschreiben Sie Ihre Vorstellung, inwiefern der kulturelle Hintergrund von Schülerinnen und Schülern einen Einfluss auf deren Lernen hat.» Oder: "Was denken Sie, was müssen Sie noch lernen, um als Lehrperson optimal auf den Umgang mit kulturell heterogenen Klassen vorbereitet zu sein?»

Um das Diagnosepotential dieses Stufenmodells nutzbar zu machen, wäre die Entwicklung eines eigentlichen Diagnose-Instrumentes hilfreich, welches das Thematisierungsvermögen bezüglich kultureller Heterogenität im schulischen Kontext zu erfassen vermag. Erste Schritte dazu haben denn auch an der Pädagogischen Hochschule Zentralschweiz vielversprechende Resultate gezeigt. Und um das Förderpotential dieses Stufenmodells nutzbar zu machen, wären stufenspezifische Entwicklungsaufgaben abzuleiten, je geeignete Unterstützungsmassnahmen zu entwickeln und entsprechende opportunities to learn (Ravitsch, 1995) zu beschreiben. Es bleibt an dieser Stelle zu hoffen, dass das Stufenmodell der Normalitätsreflexionen Inspirationen geben kann, um die Auseinandersetzung mit kultureller Heterogenität in der Lehrerinnen- und Lehrerbildung weiter zu entwickeln.

\section{Anmerkungen:}

1 Gemeint sind damit immer Phänomene, «in denen sich kulturelle, sprachliche oder soziale Verschiedenheit zeigt» (Gogolin, 2003, S. 1).

2 Wenn auch in Bennetts (1986) Developmental Model of Intercultural Sensitivity der Begriff der Reflexion nicht so explizit aufscheint wie in den anderen drei Modellen, so verweisen seine ethnorelativen Entwicklungsstadien mit der Entwicklung eines beweglichen Bezugsrahmens doch ebenso deutlich auf die Bedeutung der Reflexion. 


\section{Literaturverzeichnis}

Bender-Szymanski, D., Hesse, H.-G. \& Göbel, K. (2000). Akkulturation in der Schule: Kulturbezogene Konflikte und ihre Auswirkungen auf Denken und Handeln junger Lehrer in multikulturellen Schulen. In I. Gogolin \& B. Nauck (Hrsg.), Migration, gesellschaftliche Differenzierung und Bildung (S. 213-244). Opladen: Leske und Budrich.

Bennett, M. J. (1986). A developmental approach to training for intercultural sensitivity. International Journal of Intercultural Relations, 10 (2), 179-196.

Bruner, J. (1996). The culture of education. Cambridge, MA: Harvard University Press.

Corbin, J. (2003). Grounded Theory. In R. Bohnsack, W. Marotzki \& M. Meuser (Hrsg.), Hauptbegriffe Qualitativer Sozialforschung (S. 70-75). Opladen: Leske und Budrich.

Corbin, J. \& Strauss, A. L. (1990). Grounded Theory Research: Procedures, canons and evaluative criteria. Qualitative Sociology, 13 (1), 3-21.

Foucault, M. (1973). Archäologie des Wissens. Frankfurt a. M.: Suhrkamp.

Foucault, M. (1976). Überwachen und Strafe. Die Geburt des Gefängnisses. Frankfurt a. M.: Suhrkamp.

Gogolin, I. (1994). Der monolinguale Habitus der multikulturellen Schule. Münster: Waxmann.

Gogolin, I. (2003). Fähigkeitsstufen der interkulturellen Bildung. Hamburg: Mimeo.

Hoopes, D. S. (1979). Intercultural communication concepts and the psychology of intercultural experience. In M. D. Pusch (Hrsg.), Multicultural education: A cross-cultural training approach (S. 9-42). Yarmouth, ME: Intercultural Press.

Jahoda, G. (1996). Ansichten über die Psychologie und die «Kultur». In A. Thomas (Hrsg.), Psychologie interkulturellen Handelns (2. Auflage) (S. 33-42). Göttingen: Hogrefe.

Karakaşoğlu, Y. \& Bandorski, S. (2008). Editorial zum Schwerpunktthema «Interkulturelle Bildung». Bildungsforschung, 5 (1), 1-7.

Link, J. (2006). Versuch über den Normalismus. Wie Normalität produziert wird. Göttingen: Vandenhoeck \& Ruprecht.

Radtke, F.-O. (2004). Schule und Ethnizität. In W. Helsper \& J. Böhme (Hrsg.), Handbuch der Schulforschung (S. 625-646). Wiesbaden: VS Verlag für Sozialwissenschaften.

Ravitsch, D. (1995). National standards in american education: A citizen's guide. Washington, DC: Brookings Institution.

Reckwitz, A. (2001). Multikulturalismustheorie und der Kulturbegriff: Vom Homogenitätsmodell zum Modell kultureller Interferenzen. Berliner Journal für Soziologie, 11 (2), 179200.

Ringeisen, T., Buchwald, P. \& Schwarzer, C. (2007). Unterrichtsgestaltung aus interkultureller Perspektive. Intercultural Journal, 4, 27-48.

Strauss, A. L. (1991). Grundlagen qualitativer Sozialforschung. Datenanalyse und Theoriebildung in der empirischen soziologischen Forschung. München: Wilhelm Fink Verlag.

Thomas, A. (2003). Interkulturelle Kompetenz - Grundlagen, Probleme und Konzepte. Erwägen Wissen Ethik - Streitforum für Erwägungskultur, 14 (1), 137-228.

Ulfig, A. (1997). Lebenswelt, Reflexion und Sprache. Zur reflexiven Thematisierung der Lebenswelt Phänomenologie, Existenzialontologie und Diskurstheorie. Würzburg: Königshausen \& Neumann.

Wang, H. \& Meyers, M. J. (2007). Developing cultural awareness. In D. Neuendorff \& C. M. Schmidt (Hrsg.), Sprache, Kultur und Zielgruppen: Bedingungsgrössen für die Kommunikationsgestaltung in der Wirtschaft (S. 303-324). Wiesbaden: DUV Deutscher UniversitätsVerlag.

Schlagworte: Interkulturelle Sensibilität, Lehrerbildung, Reflexion, kulturelle Heterogenität 


\section{Un modèle par étape de réflexions sur la normalité. Les différentes positions des enseignant-e-s en matière de réflexion sur l'hétérogénéité à l'école et dans l'enseignement}

Résumé

L'évidente hétérogénéité culturelle de la société, de l'école et de l'enseignement nécessite pour la formation professionnelle des enseignants-e-s de rendre possible l'acquisition de capacités qui rendent efficace l'organisation de l'école et de l'enseignement dans un contexte multiculturel. Jusqu'ici les modèles qui conceptualisent l'acquisition de capacités interculturelles ne se centrent pas suffisamment sur le contexte spécifique de l'institution scolaire empreinte de monoculturalité. La recherche présentée ici met en évidence des modèles de réflexions prototypiques qui permettront aux futurs enseignants d'appréhender les différences culturelles dans le contexte scolaire. Il en résulte un modèle par étape des réflexions sur des normalités. Ainsi seront décrits des niveaux de réflexions en relation avec les différences culturelles dans un contexte scolaire monoculturel qui pourront être diagnostiquées puis traitées de manière adéquate dans la formation des enseignant-e-s.

Mots clés: Sensibilité interculturelle, formation des enseignants, réflexivité, hétérogénéité culturelle.

\section{Un modello graduale di riflessioni sulla normalità. He differenti posizioni degli insegnanti al riguardo dell'eterogeneità culturale a scuola e nell'insegnamento}

\section{Riassunto}

L'evidente eterogeneità della società, della scuola e dell'insegnamento richiede, nel percorso di formazione professionale degli insegnanti, l'acquisizione di capacità che rendano efficace l'organizzazione della scuola e dell'insegnamento in un contesto multiculturale. Fino ad oggi i modelli che concettualizzano l'acquisizione di capacità interculturali non focalizzano sufficientemente il contesto specifico dell'istituzione scolastica improntata alla monoculturalità. Questa ricerca evidenzia dei modelli di riflessioni prototipiche in grado di permettere ai futuri insegnanti di afferrare le differenze culturali nel contesto scolastico. Ne risulta un modello graduale di riflessioni sulla normalità. Saranno così descritti diversi livelli di riflessioni in relazione con le differenze culturali in un contesto scolastico monoculturale, che potranno quindi essere diagnosticate e poi adeguatamente trattate nella formazione degli insegnanti.

Parole chiave: Sensibilità interculturale, formazione degli insegnanti, riflessività, eterogeneità culturale 


\title{
Stage model of normality reflections. How teachers reflect differently on cultural heterogeneity in the school context
}

\begin{abstract}
Today's prevalent cultural heterogeneity in society, schooling and teaching poses a challenge to teacher education to provide teachers with the necessary skills to enable them to develop productive measures for schooling and teaching in a multicultural context. Previous models which conceptualise the acquisition of intercultural skills, however, focus too little on the specific context of predominantly monocultural schooling. The research project presented in this paper has thus designed prototypical reflection examples as to how (future) teachers could reflect on cultural differences specific to the school context and incorporates them into a stage model of normality reflections. In this way, different levels of reflection regarding cultural differences in monocultural school contexts are described which can be used for diagnostic purposes and further developed in teacher education.
\end{abstract}

Key words: Intercultural sensitivity, teacher education, reflection, cultural diversity 